American Journal of Biochemistry and Biotechnology 4 (2): 121-129, 2008

ISSN 1553-3468

(C) 2008 Science Publications

\title{
Peripheral Blood Leukocyte Production of BDNF following Mitogen Stimulation in Early Onset and Regressive Autism
}

\author{
${ }^{1}$ Amanda Enstrom, ${ }^{1}$ Charity Onore, ${ }^{1}$ Angela Tarver, ${ }^{2}$ Irva Hertz-Picciotto, ${ }^{3}$ Robin Hansen, ${ }^{4}$ Lisa Croen, \\ ${ }^{5}$ Judy Van de Water and ${ }^{1}$ Paul Ashwood \\ ${ }^{1}$ Department of Medical Microbiology and Immunology and the M.I.N.D. Institute \\ University of California at Davis \\ ${ }^{2}$ Department of Public Health Sciences, Division of Epidemiology, University of California at Davis \\ ${ }^{3}$ Department of Pediatrics, University of California at Davis and M.I.N.D. Institute \\ ${ }^{4}$ Division of Research, Kaiser Permanente Northern California, Oakland, CA \\ ${ }^{5}$ Division of Rheumatology, Allergy and Clinical Immunology, University of California at Davis
}

\begin{abstract}
Brain-derived neurotrophic factor (BDNF) is critical for neuronal differentiation and synaptic development. BDNF is also implicated in the development of psychological disorders including depression, bipolar disorder and schizophrenia. Previously, elevated BDNF levels were observed in neonatal blood samples from infants who were later diagnosed with autism when compared with children who developed normally, suggesting that BDNF may be involved in the development of autism. BDNF is produced by activated brain microglial cells, a cellular phenotype that shares several features with peripheral macrophages, suggesting an important role for the immune system in BDNF production. We hypothesized that under mitogenic stimulation, peripheral blood mononuclear cells obtained from children with autism may have altered BDNF production compared with age-matched typically developing control subjects. In addition, we examined the differences between the production of BDNF in classic/early-onset autism and children who had a regressive form of autism. We show here that plasma levels of BDNF levels are increased in children with autism, especially in early onset autism subjects. Furthermore, under mitogenic stimulation with PHA and LPS, BDNF production is significantly increased in children with autism compared with typically developing subjects. However, stimulation with tetanus toxoid results in a decreased response in children with autism. This data suggest that immune cell-derived production of BDNF could be an important source for the increased BDNF that is detected in some subjects with autism. As a neurotrophic factor produced by immune cells, BDNF could help elucidate the role of the immune system in neurodevelopment and neuronal maintenance, which may be dysregulated in autism.
\end{abstract}

Key words: Brain-derived neurotrophic factor (BDNF), luminex, neurotrophin, autism, inflammation

\section{INTRODUCTION}

Autism is a complex neurodevelopmental disorder that appears within the first three years of life and is characterized by significant impairments in social interaction and communicative skills. Most cases of autism present classically, with the affected child failing to develop normal social or language skills, which is termed here as early onset autism. However, in a subset of cases, known as regressive autism, the child appears to develop typically until approximately 18-24 months of age, when the child loses many previously acquired social and language skills ${ }^{[1]}$. In general, the etiology of autism remains elusive, with many factors implicated in the development of the autism phenotype.
There is a strong heritable component of autism based on family and twin studies. Furthermore, co-morbidity exists between family members of subjects with autism and affective disorders, especially depression ${ }^{[2-4]}$. Several genetic studies link autism with genes that have immune functions such as HLA, Complement C4, MET, REELIN and recently, a region on chromosome 11 p12-p13 that encodes many important immune related genes ${ }^{[5-9]}$. However, genetic studies have not yet revealed a specific definitive autism marker which has led to the hypothesis that an environmental trigger could be associated with some cases of autism. Heavy metal exposure, vaccination and perinatal infection at key neurodevelopmental junctures ${ }^{[10-12]}$, have all been hypothesized to be involved in the development of

Corresponding Author: $\quad$ Dr. Paul Ashwood, Department of Microbiology and Immunology and the M.I.N.D. Institute, $280550^{\text {th }}$ Street, Wet lab building, Sacramento, CA 95817 
autism but their effects remain widely debated. It is also likely that any environmental trigger could vary with a particular individual and influence neurodevelopment in specific ways dependent upon the genetic susceptibility of that individual. Alterations in neurodevelopment in the fetus or early in life could result in altered brain development which may ultimately lead to features characteristic of autism. A large, growing body of research has revealed that immune responses can influence neurodevelopment and that significant alterations in the immune system may play a key role in some individuals with autism ${ }^{[13]}$.

BDNF is important for neurodevelopment, neuronal differentiation and neuronal protection. Early in development, microglial cells, a cellular phenotype that share several similarities with peripheral macrophages, are evident within neuronal tissue. Activated microglial cells are able to secrete BDNF, which may protect the brain from inflammationinduced apoptosis and may alter synaptic properties of the neurons ${ }^{[14,15]}$. Deviations in BDNF levels have been linked to deficits in serotonin, which is associated with mood disorders. In at least a subset of autism, altered serotonin levels are among one of the few consistent research findings ${ }^{[16-18]}$. BDNF has been associated with changes in behavior, such as hyperactivity, increased depression and psychiatric disorders including schizophrenia and bipolar disorder ${ }^{[19-20]}$. Decreased plasma BDNF levels correspond strongly with severity of symptoms ${ }^{[21,22]}$. In a murine model, Branchi et al. reported that lower BDNF levels directly equated to changes in social aptitude, anxiety and aggression ${ }^{[23]}$.

Multiple studies have shown increases in BDNF in both blood and brain tissue from autistic subjects ${ }^{[24-26]}$. Since BDNF readily crosses the blood brain barrier, serum concentrations correlate directly to brain concentrations; therefore plasma studies of BDNF are thought to accurately reflect CNS concentrations ${ }^{[27-28]}$. In a retrospective study on blood obtained from fullterm neonates, Nelson et al. identified a nearly threefold increase in BDNF levels in children later diagnosed with autism spectrum disorder compared with typically developing children ${ }^{[25]}$. This study is especially revealing since the results were obtained early, before symptoms appeared; although a later subsequent study by the same group failed to confirm these results ${ }^{[29]}$. In animal studies, maternal infection has been shown to significantly increase BDNF levels in the fetal brain ${ }^{[30,31]}$. The affect on BDNF production following maternal infection with lipopolysaccharide (LPS), a component of gram negative bacterial cell walls, suggests an immune mechanism for altered neurotrophin production ${ }^{[30]}$. Maternal infections and exposure to LPS have been directly tied to behavior and neurodevelopmental changes in animal models that may be applicable to autism ${ }^{[32-36]}$. In addition, BDNF expression was shown to be increased three-fold in the forebrains of adults with autism when compared with brain specimens from control adults ${ }^{[26]}$, suggesting that alterations in BDNF expression in autism may be important throughout the life of the individual.

Changes in BDNF levels have been linked to a number of neurologic disorders, including autism, however little is known on the mechanism behind these altered levels in autism ${ }^{[14,21,22,24-26,37]}$. BDNF is produced by activated immune microglial cells $\mathrm{s}^{[14,15]}$ and may directly act on astrocytes to alter neuronal transmission. Indeed, postmortem analysis of brain material from subjects with autism revealed not only marked activation of microglia but also astroglia ${ }^{[38]}$. In vitro assays have demonstrated that BDNF stimulates neuronal outgrowth on neonatal astrocytes ${ }^{[39]}$, which may explain, in part, previous findings of larger neuronal cells in young subjects with autism ${ }^{[40]}$. Interestingly, in animal models maternal viral infection has been linked to macrocephaly in the offspring ${ }^{[41]}$. It is possible that following an immune challenge, BDNF may be changed potentially leading to an altered course of neurodevelopment that could result in significant disturbances. Experimental evidence points to proinflammatory activity in some autism subjects that could lead to the aberrant production of BDNF from immune cells such as monocytes and B cells. To help elucidate the role of an immune challenge on BDNF production by immune cells, we stimulated peripheral blood cells using various immune cell mitogens, including phytohemagglutinin (PHA), gram-negative bacterial membrane component LPS and tetanus toxoid. Despite previous evidence of increased BDNF levels in individuals with autism, differential BDNF production in subsets of autism, based on onset of behavior, has not been examined but may help illuminate discrepancies in prior findings ${ }^{[25,29]}$. Therefore, in addition, we examined whether various immune mitogens could differentially induce BDNF production by immune cells in children with early onset autism compared with regressive autism and typically developing control children.

\section{MATERIALS AND METHODS}

Subjects: Participants in the study were recruited in conjunction with the CHARGE (Childhood Autism Risk from Genetics and Environment) study conducted at the UC Davis M.I.N.D. Institute ${ }^{[42]}$. Participants included 125 children ranging from 2-5 years of age 
who met one of the following 4 criteria: 1) diagnosed with autism; 2) diagnosed with developmental delay but not autism; 3) an unaffected sibling of a child with autism; or 4) general population controls. The descriptive statistics of the study population are described in Table 1 . This study was approved by the UC Davis institutional review board and complied with all requirements regarding human subjects. Informed consent was obtained from a legal guardian of each participant. To confirm diagnosis, all autism participants completed the Autism Diagnostic Interview-Revised (ADI-R $)^{[43]}$ and the Autism Diagnostic Observation Schedule (ADOS) ${ }^{[43-46]}$ which were administered at the M.I.N.D. Institute by qualified trained clinicians. The ADI-R is a comprehensive clinical interview administered to parents or caregivers of children suspected of having autism. The ADI-R assesses function in areas of language and communication, reciprocal social interaction and repetitive, restricted, stereotyped behaviors or interests. Results from the interview are interpreted by using a diagnostic algorithm that correlates with the DSM-IV and ICD-10 definitions of autism. The ADOS is an observational assessment of the children suspected of having autism in various structured and unstructured situations that provide a standardized set of conditions to observe behavior in the areas of communication, play and other areas relevant to autism. Children from the developmental delay and general population groups were screened for autism traits using the Social Communication Questionnaire. The ADI-R and ADOS was administered to those scoring above the screening threshold to determine autism status. Children who scored above the cut-off for the ADOS modules 1 and 2 and met the criteria for autism in the ADI-R were included in the autism group. Children confirmed as autism subjects were divided into subgroups of early onset or regressive autism based on the stage of development the child began to display autistic behavior. Children were classified as having early onset autism if the child exhibited traits of autism from infancy and regressive autism if they had typical early development and later lost function in the areas relevant to autism based on ADI-R scores.

Peripheral Blood Mononuclear Cell (PBMC) Isolation: Peripheral blood was collected in acid- citrate-dextrose Vacutainers (BD Biosciences; San Jose, $\mathrm{CA}$ ), centrifuged at $2300 \mathrm{rpm}$ for $10 \mathrm{~min}$ and plasma removed from sample. Blood cell suspension was adjusted to a volume of $20 \mathrm{~mL}$ with Hank's Balanced Salt Solution (HBSS) (VWR; West Chester, PA), layered on room temperature Histopaque (Sigma; St. Louis, MO) and centrifuged at $1700 \mathrm{rpm}$ for $30 \mathrm{~min}$ to isolate PBMCs. PBMCs were extracted and washed twice with HBSS and the number of viable PBMCs was determined by Trypan Blue exclusion (Sigma, St. Louis, MO).

PBMC Mitogen Stimulation: PBMCs were adjusted to a concentration of $3.0 \times 10^{6}$ cells mL $\mathrm{mL}^{-1}$ with a solution of $0.2 \%$ T-Stim (BD Biosciences) in serum-free X-Vivo media (Cambrex, Walkersville, MD). For each subject, $100 \mu \mathrm{L}$ of the PBMC suspension was plated in four individual wells of a 96-well tissue culture plate (Corning, Corning, NY). $100 \mu \mathrm{L}$ of stimulant or media was added to each well for a final volume of $200 \mu \mathrm{L}$. The PBMCs were stimulated with $2 \mu \mathrm{g}$ phytohemagglutinin (PHA; Sigma), $5 \mu \mathrm{g}$ lipopolysaccharide (LPS; Sigma), 0.25 LF (limit of flocculation) units Tetanus Toxoid (Cylex, Columbia, $\mathrm{MD})$, or $100 \mu \mathrm{L} \mathrm{X}$-Vivo alone. All antigen dilutions were made in X-Vivo. The cells were incubated at $37^{\circ} \mathrm{C}$ for $48 \mathrm{hrs}$ at which time the plates were centrifuged and supernatants from the four stimulation conditions were individually harvested by pipette and stored at $-80^{\circ} \mathrm{C}$ until the date of assay.

BDNF Concentration Measurement: Plasma and PBMC culture supernatants were analyzed by luminex using the Human BDNF Antibody Bead Kit (Biosource, Camarillo, CA). The assay kit allowed for BDNF detection between $35-7600 \mathrm{pg} \mathrm{mL}^{-1}$. Analyte specific antibody conjugated beads were incubated with $50 \mu \mathrm{L}$ of $1 \mathrm{x}$ biotinylated detection antibody solution and 50 $\mu \mathrm{L}$ of either standard, or $25 \mu \mathrm{L}$ of sample in $25 \mu \mathrm{L}$ of assay diluent for $3 \mathrm{hrs}$ at room temperature, in the dark, on an orbital shaker (500-600 rpm). After wells were washed and aspirated by vacuum manifold aspiration, $100 \mu \mathrm{L}$ of strepavidin-conjugated R-phycoerythrin was added to each well and incubated for $30 \mathrm{~min}$ at room temperature, in the dark, on an orbital shaker (500-600 rpm). R-phycoerythrin fluorescence was measured on a Bio-Plex 200 System (Bio-Rad Laboratories Inc., Hercules, CA) to determine concentration. For PHA, LPS and tetanus-toxoid supernatants, the concentration of the media alone was subtracted from the value to analyze BDNF secretion specific to the stimulant. 
Statistical analysis: Student's two-tailed t-test for samples with unequal variances was used to examine the data for statistical significance. Values were considered significant for $\mathrm{p}<0.05$.

\section{RESULTS AND DISCUSSION}

Plasma BDNF levels: Initially we examined BDNF protein levels in the plasma of the subjects (Fig. 1). Plasma BDNF levels in children with autism (2512.103 $\pm 327.79 \mathrm{pg} \mathrm{mL}^{-1}$, mean \pm S.E.M.) were significantly increased compared with age-matched non-autistic control subjects $\left(1463.07 \pm 174.61 \mathrm{pg} \mathrm{mL}^{-1}, \mathrm{p}=0.043\right.$, Fig. 1A). Within the autism group, children with early onset autism had a non-statistically significant trend towards higher BDNF levels $(2836.36 \pm 526.82 \mathrm{pg}$ $\mathrm{mL}^{-1}$ ) when compared with children with regressive autism $(2130.62 \pm 333.89$, Fig. $1 \mathrm{~B}, \mathrm{p}>0.05)$. As a consequence, subjects with early onset autism had statistically significantly higher plasma BDNF levels compared with typically developing children $(\mathrm{p}=0.032)$, siblings of children with autism $(\mathrm{p}=0.019)$ and children with MR/DD ( $\mathrm{p}=0.023)$. However, plasma BDNF levels did not reach statistical significance for the regressive autistic children compared with typically developing controls $(\mathrm{p}=0.06)$. There were no differences in plasma BDNF levels between typically developing control children $(1486.91+147.89$ pg $\mathrm{mL}^{-1}$ ), children with mental retardation and/or developmental delay (MR/DD) $(1457.59 \pm 211.09 \mathrm{pg}$ $\left.\mathrm{mL}^{-1}\right)$ and siblings of children with autism $(1444.72 \pm$ $164.84 \mathrm{pg} \mathrm{mL}^{-1}$, Fig. 1C, p>0.05).

BDNF production by cultured peripheral blood mononuclear cells (PBMC) following stimulation: Similar to results seen in the plasma blood compartment, isolated non-stimulated PBMC from children with autism secreted more BDNF $(46.24 \pm$ $9.19 \mathrm{pg} \mathrm{mL}^{-1}$ BDNF, mean \pm S.E.M) in a $48 \mathrm{hr}$ period compared with age-matched typically developing control children $\left(36.12 \pm 6.50 \mathrm{pg} \mathrm{mL}{ }^{-1}\right.$ BDNF, $\mathrm{p}=$ 0.043 , Fig. 2A). Unstimulated BDNF values did not differ between children with early onset autism (45.99 $\pm 8.62 \mathrm{pg} \mathrm{mL}^{-1}$ ) and children with regressive autism $\left(46.49 \pm 9.75 \mathrm{pg} \mathrm{mL}^{-1}, \mathrm{p}>0.05\right.$, Fig. $\left.2 \mathrm{~B}\right)$, although there was great variability amongst the children with regressive autism, ranging from 5.51 to $120.70 \mathrm{pg} \mathrm{mL}^{-1}$ BDNF.

BDNF production from PBMC under different stimulation treatment conditions are shown in Table 1. Children with autism produced more BDNF following stimulation with the mitogen PHA $(58.00 \pm 9.46 \mathrm{pg}$ $\mathrm{mL}^{-1}$, mean \pm S.E.M.) compared with typically developing age-matched control children $(42.47 \pm 7.47$ pg mL ${ }^{-1}, \mathrm{p}=0.050$, Table 2). No statistically significant differences in BDNF production were seen between cases and controls following stimulation with LPS or
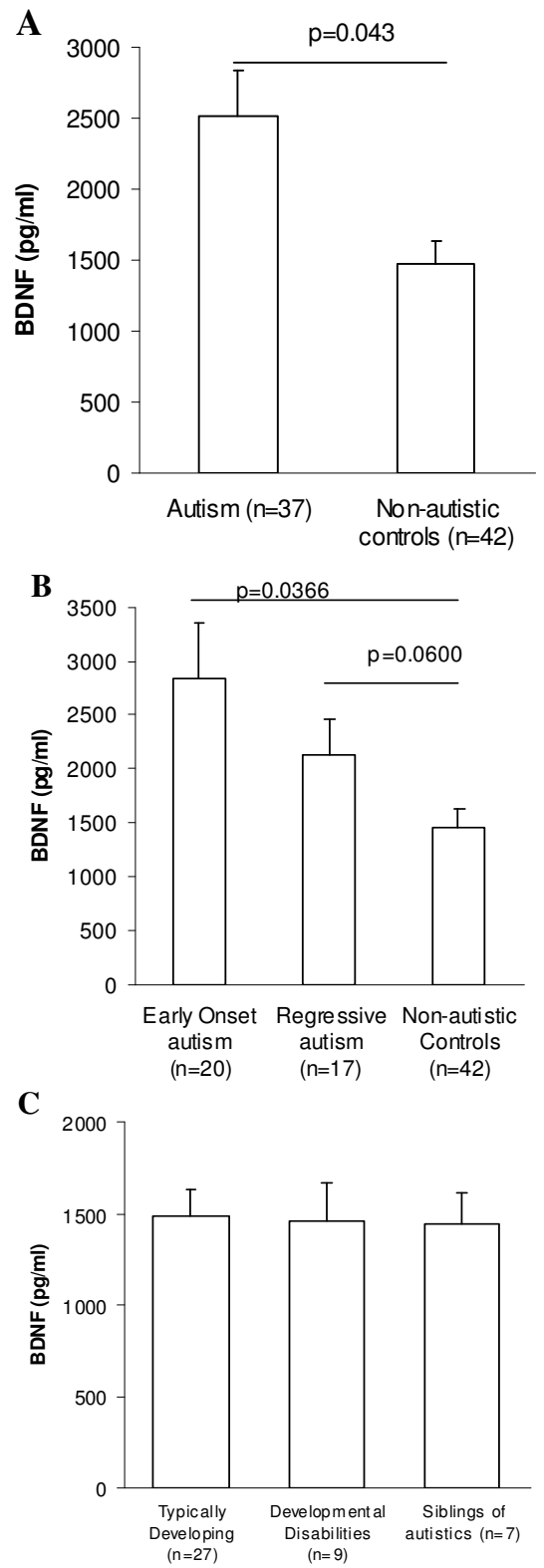

Fig. 1: BDNF protein levels in plasma. (A) Comparison of BDNF levels between subjects with autism and non-autism control subjects. Children with autism had higher mean plasma BDNF levels compared with subjects without autism. (B) BDNF levels are compared between subjects with early onset autism, regressive autism and control subjects. Subjects with early onset autism had higher plasma BDNF levels compared with control subjects and a trend to elevated levels compared with subjects with regressive autism. (C) Typically developing children, children with developmental disabilities and siblings of children with autism had similar BDNF protein levels in plasma 
Am. J. Biochem. \& Biotech., 4 (2): 121-129, 2008
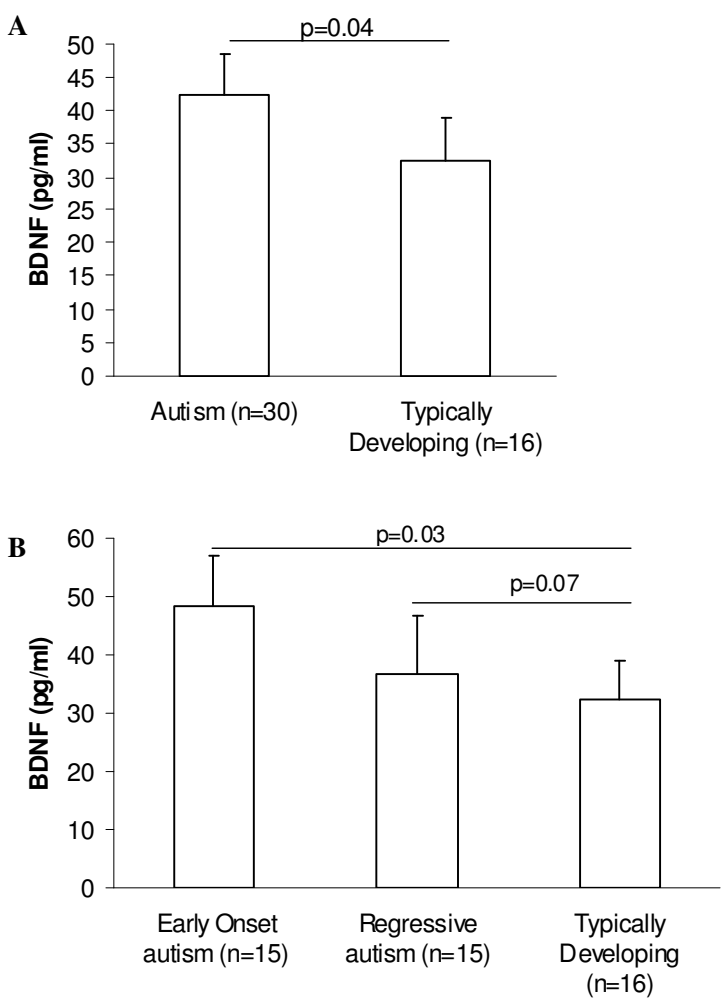

Fig. 2: Production of BDNF by cultured PBMC. $3.0 \times 10^{5}$ PBMC were cultured for $48 \mathrm{hrs}$ in $100 \mu \mathrm{L} \mathrm{X}$-Vivo media without mitogen. Cells from subjects with (A) autism produced more BDNF during this period than typically developing controls; moreover (B) higher BDNF was produced from cells obtained from subjects with early onset autism rather than regressive autism. P-values are shown using Student's twotailed t-test for samples with unequal variances

tetanus toxoid $(\mathrm{p}>0.05)$. When the autism population was separated based on onset of behavioral criteria, PBMC from early onset and regressive autism children responded differently following stimulation. Following PHA stimulation BDNF production was increased significantly in early onset autism children but not autism children with regression compared with typically developing controls $(\mathrm{p}<0.036$, Table 2$)$. Furthermore, following baseline correction with media alone values, children with early onset autism produced significantly increased levels of BDNF $(13.12+12.06$ pg $\mathrm{mL}^{-1}$, mean change above media baseline + S.E.M.), when stimulated with PHA compared with regressive autism children $\left(4.50 \pm 2.48 \mathrm{pg} \mathrm{L}^{-1} 1, \mathrm{p}=0.048\right.$, Fig. 3$)$ stimulated under the same conditions. There were also decreased BDNF responses in children with regressive autism but not early onset autism following stimulation with LPS $\left(5.35 \pm 4.59 \mathrm{pg} \mathrm{mL}^{-1}\right)$ compared with agematched typically developing children $(10.67 \pm 2.31 \mathrm{pg}$ $\mathrm{mL}^{-1}, \mathrm{p}=0.045$, Fig. 3). Similarly, when stimulated with

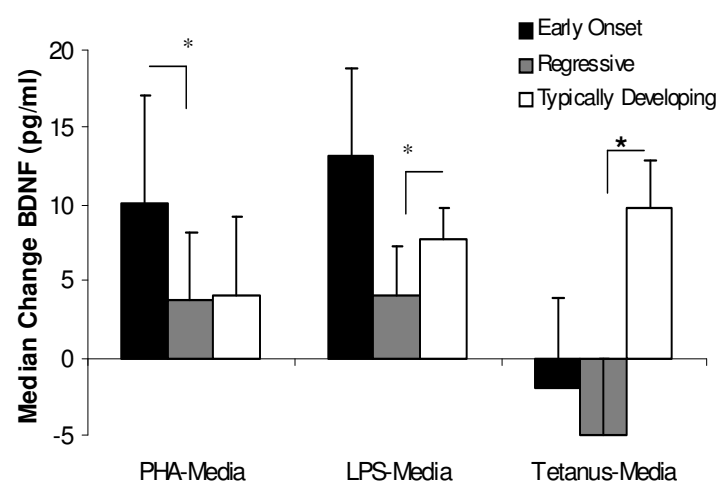

Fig. 3: BDNF production by PBMC following mitogen stimulation. $3.0 \times 10^{5}$ PBMC were stimulated for $48 \mathrm{~h}$ in $200 \mu \mathrm{L}$ final volume containing $2 \mu \mathrm{g}$ phytohemagglutinin (PHA), $5 \mu \mathrm{g}$ lipopolysaccharide (LPS), or 0.25 LF units tetanus toxoid. Amount of BDNF produced from control wells stimulated with media alone was subtracted from results to examine effects of stimulant alone $\left(^{*} \mathrm{p}<0.05\right)$

tetanus-toxoid, PBMC from children with regressive autism but not early onset autism secreted decreased levels of BDNF than with media alone $(-5.22+3.8 \mathrm{pg}$ $\mathrm{mL}^{-1}$ ) and were significantly reduced compared with tetanus-toxoid stimulated responses in typically developing controls $(3.18 \pm 3.29, \mathrm{p}=0.046$, Fig. 3$)$.

This study is the first to look at differential BDNF expression by stimulated lymphocytes in autism. It is also the first to examine if there is a differential response in BDNF based on behavioral patterns in autism. Our finding that BDNF levels are increased in the plasma of autistic children is consistent with previous reports of BDNF in autism ${ }^{[24-26]}$. Moreover, we noted that plasma BDNF may be different in autism children based on patterns of onset, with a trend to higher levels in children with early onset autism. We found no differences in BDNF levels when comparing amongst typically developing subjects, siblings of children with autism and mental retardation/ developmentally delayed children. Previous reports have shown that in mental retardation the levels of BDNF are more similar to autism than compared with normal controls ${ }^{[25]}$. In contrast, our findings suggest that the increased BDNF may relate to an autism diagnosis specifically and not to a more general neurodevelopmental dysfunction. Furthermore, since siblings of children with autism likely share several genetic polymorphisms with their affected sibling, it was anticipated that BDNF in these subjects would be different to levels of the general population typically developing controls. However, as BDNF was not dysregulated in the siblings of children with autism, the alterations in BDNF levels seen in the autism population may be attributed specifically to the autism 
Am. J. Biochem. \& Biotech., 4 (2): 121-129, 2008

Table 1: Descriptive statistics of the study population

\begin{tabular}{|c|c|c|c|c|c|c|}
\hline & \multicolumn{3}{|l|}{ Autism } & \multirow{2}{*}{$\begin{array}{l}\text { Typically } \\
\text { Developing }\end{array}$} & \multirow{2}{*}{$\begin{array}{l}\text { Siblings } \\
\text { of autism }\end{array}$} & \multirow{2}{*}{$\begin{array}{l}\text { Developmental } \\
\text { Disabilities }\end{array}$} \\
\hline & Total & Regressive & Early Onset & & & \\
\hline \multicolumn{7}{|l|}{ Plasma BDNF } \\
\hline $\mathrm{n}=$ & 37 & 17 & 20 & 27 & 9 & 6 \\
\hline$\%$ Males & 91 & 94 & 89 & 67 & 56 & 67 \\
\hline Median Age & 3.58 & 3.25 & 4.04 & 3.13 & 3.83 & 2.71 \\
\hline Age Range & $2.08-5.92$ & $2.08-4.83$ & $2.17-5.92$ & $2.17-4.58$ & $2.25-4.58$ & $2.0-3.42$ \\
\hline \multicolumn{7}{|c|}{ Cellular Stimulation Assays } \\
\hline $\mathrm{n}=$ & 30 & 15 & 15 & 16 & $n / a$ & $n / a$ \\
\hline$\%$ Males & 90 & 93 & 87 & 89 & & \\
\hline Median Age & 3.54 & 3.33 & 4.08 & 3.67 & & \\
\hline Age Range & $2.33-5.17$ & $2.5-5.17$ & $2.33-5.33$ & $1.5-4.92$ & & \\
\hline
\end{tabular}

Table 2: Total BDNF present in supernatants of unstimulated PBMC (media) and PBMC stimulated with phytohemagglutinin (PHA),

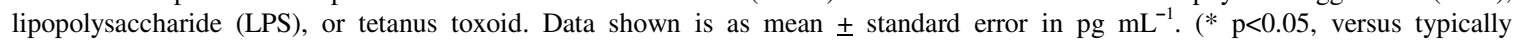
developing subjects)

\begin{tabular}{lcccc}
\hline & Total Autism Subjects & Early onset Autism & Regressive Autism & Typically Developing \\
\hline Media & $46.24 \pm 6.39^{*}$ & $45.99 \pm 8.62^{*}$ & $46.49 \pm 9.75$ & $36.12 \pm 6.50$ \\
PHA & $58.00 \pm 9.46^{*}$ & $59.11 \pm 9.79^{*}$ & $56.88 \pm 16.58$ & $37.95 \pm 7.47$ \\
LPS & $49.17 \pm 6.20$ & $46.50 \pm 8.95$ & $51.84 \pm 8.84$ & $42.60 \pm 8.68$ \\
Tetanus & $42.57 \pm 5.25$ & $44.66 \pm 8.66$ & $40.49 \pm 6.23$ & $35.72 \pm 5.85$ \\
\hline
\end{tabular}

phenotype. We note that the small sample size may affect the interpretation of these specific comparisons and these findings should be confirmed in a larger study.

Changes in BDNF expression have been linked to mood disorders and neuronal dysregulation ${ }^{[14,15,19,20,}$ 23]. Neonatal blood testing suggests that BDNF expression may be aberrant at birth in autism, which may implicate a possible role in the prenatal development of the autism phenotype ${ }^{[25]}$. In addition, in a study of adult forebrains, increased BDNF in subjects with autism persisted through adulthood ${ }^{[37]}$, suggesting as well that any detrimental neuronal effects of increased BDNF may persist throughout the life of the individual. Since BDNF has been shown to be secreted by activated microglial cells ${ }^{[14,15]}$, we hypothesized that another cellular source for the increased BDNF levels may arise from peripheral leukocytes. In this study we quantified the production of BDNF from leuokocytes stimulated with; PHA, a plant lectin which broadly activates lymphocytes; LPS, a gram-negative bacterial membrane component; and tetanus toxoid, produced by the gram-positive bacteria Clostridium tetani. The use of three different treatment conditions allowed us to make a preliminary examination of the cellular BDNF response during activation with different types of potential pathogens, such as may occur during an infectious exposure. Children with autism had increased BDNF production when stimulated with PHA and LPS compared with typically developing children, but not when stimulated with tetanus toxoid. This is interesting, as tetanus toxoid action on lymphocytes is restricted in this experimental system and follows antigen recognition via $\mathrm{MHC}$ molecules present on antigen presenting cells, in contrast, PHA and LPS can directly act on lymphocytes. It is tempting to suggest that altered antigen presentation in autism may lead to the apparent reduction in BDNF seen following tetanustoxoid stimulation. Furthermore, differences in BDNF levels following stimulation were observed based on behavioral patterns in autism. In general, there was a trend throughout for an elevated BDNF response in children with early onset autism when compared with regressive autism, over all treatment conditions. In addition, children with autism produced more BDNF in non-stimulatory conditions compared with typically developing controls, suggesting that there may be an increased constitutive expression of BDNF in autism that is further stimulated in the presence of certain pathogenic or infectious insults, a finding that may be more apparent in children with early onset autism. Since BDNF has significant impact on neuronal functioning in the brain, this upregulated response may be important in some of the key features of autism.

Aberrant neuroimmune cross-talk could precipitate neuronal damage or improper neurotrophic signaling. Further evidence of altered immune activation in autism includes proinflammatory cytokines, including $\mathrm{TNF} \alpha$, which has been shown to be increased in plasma and blood lymphocytes of autistic individuals ${ }^{[47-49]}$. These proinflammatory cytokines have been linked with increased secretion of neurotrophic factors ${ }^{[47-52]}$, which are critical regulators of synaptic development and 
maintenance, as well as neuron survival and differentiation ${ }^{[33,54]}$. In response to administered proinflammatory cytokines IL-6 and TNF $\alpha$, but not to Th1 and Th2 cytokines, BDNF expression is induced in human monocytes ${ }^{[50]}$. Therefore, the upregulation of proinflammatory cytokines in autism could correlate with altered levels of neurotrophic factors, the source for which could be peripheral immune cells. In multiple sclerosis (MS), a demyelinating autoimmune disease, research has demonstrated that increased BDNF levels in this disease, at least in part, is due to secretion by activated microglial and $\mathrm{T}$ cells at the sites of the lesions ${ }^{[37]}$. Previous infection and/or immune challenge, has long been thought to be one of the putative initiating factors in the neuroinflammation that leads to both microglial and $\mathrm{T}$ cell activation in $\mathrm{MS}^{[55,56]}$.

In summary, we were able to detect differential plasma levels of BDNF in autism subjects compared with age-matched typically developing control children. Based on patterns of regression and the onset of autism behaviors, we found that in early onset autism, increased BDNF production was evident in both the plasma and in peripheral blood leukocytes following mitogenic stimulation, when compared with children who had regressive autism. This is particularly compelling as it could be suggestive of a more pronounced BDNF dysregulation in early onset autism that potentially alters neurodevelopment in this autism subset. This research suggests, for the first time, that there may be aberrant BDNF production by peripheral leukocytes in autism compared with typically developing age-matched control children and importantly, that within the autism population there is a differential BDNF response based on the onset of their behaviors. Further research would help to illuminate the role of leukocyte-derived BDNF production in the development of autism. As a neurotrophin produced, in part, by immune cells, BDNF could link the dysregulation seen in both the neurological and immunogical systems in autism.

\section{ACKNOWLEDGEMENTS}

We would like to thank the children and families who participated in this study. We also would like to acknowledge the staff of the UC Davis M.I.N.D. Institute and CHARGE study for their technical support and expertise. This work was funded by grants from NIEHS Children's Center grant (1 P01 ES11269-01), US EPA STAR program grant (R829388), the Cure
Autism Now Foundation, the Ted Lindsay Foundation and a generous gift from the Johnson family.

\section{REFERENCES}

1. Goldberg, W., K. Osann, P. Filipek, T. Laulhere, K. Jarvis, C. Modahl, P. Flodman and M.A. Spence, 2000. Language and other regression: assessment and timing. J. Autism Develop. Disord., 33: 607-616.

2. Murphy, M., P.F. Bolton, A. Pickles, E. Fombonne, J. Piven and M. Rutter, 2000. Personality traits of the relatives of autistic probands. Physcholog.1 Med., 30: 1411-1424.

3. Micali, N., S. Chakrabarti and E. Fombonne, 2004. The broad autism phenotype - finding from an epidemiological survey. Autism, 8: 21-37.

4. Yirmiya, N. and M. Shaked, 2005. Psychiatric disorders in parents of children with autism: A meta-analysis. J. Child Physchol. Physch., 46: 6983.

5. Polleux, F. and J.M. Lauder, 2004. Toward a developmental neurobiology of autism. Ment. Retard. Dev. Disabil. Res. Rev., 10: 303-317.

6. Burger, R.A. and R.P. Warren, 1998. Possible immunogenetic basis for autism. Ment. Retard. Dev. Disabil. Res. Rev., 4: 137-141.

7. Campbell, D.B., J.S. Sutcliffe, P.J. Ebert, R. Milterni, C. Bravacci, S. Trillo, M. Elia, C. Schneider, R. Melmed, R. Sacco, A.M. Perisco and P. Levitt, 2006. A genetic variant that disrupts MET transcription is associated with autism. Proc. Natl. Acad. Sci., 103: 16834-16839.

8. Skaar, D.A., Y. Shao, J.L. Haines, J.E. Stenger, J. Jaworski, E.R. Martin, G.R. DeLong, J.H. Moore, J.L. McCauley, J.S. Sutcliffe, A.E. Ashley-Koch, M.L. Cuccaro, S.E. Folstein, J.R. Gilbert and M.A. Pericak-Vance, 2005. Analysis of the RELN gene as a genetic risk factor for autism. Mol. Psychiatry, 10: $563-571$.

9. The autism project consortium, 2007. Mapping autism risk loci using genetic linkage and chromosome rearrangements. Nat. Gen., 39: 319328.

10. Mutter, J., J. Naumann, R. Schneider, H. Walach and B. Haley, 2005. Mercury and autism: accelerating evidence? Neuroendocrinol. Lett., 26: 439-446.

11. Windham, G., L. Zhang, R. Gunier, L.A. Croen and J.K. Grether, 2006. Autism spectrum disorders in relation to distribution of hazardous air pollutants in the San Francisco Bay Area. Environ. Health Perpect., 114: 1438-1444. 
12. Libbey, J.E., T.L. Sweeten, W.M. McMahon and R.S. Fujinami, 2005. Autistic disorder and viral infections. J. Neurovirol. 11: 1-10.

13. Ashwood, P., S. Wills and J. Van De Water, 2006. The immune response in autism: a new frontier for autism research. J. Leukocyte Biol., 80: 1-15.

14. Bessis, A., C. Bechade, D. Bernard and A. Roumier, 2007. Microglial control of neuronal death and synaptic properties. Glia, 55: 233-238.

15. Besser, M. and R. Wank, 1999. Cutting edge: clonally restricted production of the neurotrophins brain-derived neurotrophic factor and neurotrophin- 3 mRNA by human immune cells and Th1/Th2-polarized expression of their receptors. J. Immunol., 162: 6303-6306.

16. Cook, E.H., R. Courchesne, C. Lord, N.J. Cox, S. Yan, A. Lincoln, R. Haas, E. Courchesne and B.L. Leventhal, 1997. Evidence of linkage between the serotonin transporter and autistic disorder. Mol. Psychiatry, 2: 247-250.

17. Devlin, B., E.H. Cook, H. Coon, G. Dawson, E.L. Grigorenko, W. McMahon, N. Minshew, D. Pauls, M. Smith, M.A. Spence, P.M. Rodier, C. Stodgell and G.D. Schellenberg, 2005. Autism and the serotonin transporter: The long and short of it. Mol. Psychiatry, 10: 1110-1116.

18. Connors, S.L., K.J. Matteson, G.A. Sega, C.B. Lozzio, R.C. Carroll and A.W. Zimmerman, 2006. Plasma serotonin in autism. Ped. Neurol., 35: 182186.

19. Monteggia, L.M., B. Luikart, M. Barrot, D. Theobold, I. Malkovska, S. Nef, L.F. Parada and E.J. Nestler, 2007. Brain-derived neurotrophic factor conditional knockouts show gender differences in depression-related behaviors. Biol. Psychiatry, 61: 187-197.

20. Chan, J.P., T.J. Unger, J. Byrnes and M. Rios, 2006. Examination of behavioral deficits triggered by targeting BDNF in fetal or postnatal brains of mice. Neuroscience, 142: 49-58.

21. Shimizu, E., K. Hashimoto, N. Okamura, K. Koike, N. Komatsu, C. Kumakiri, M. Nakazato , H. Watanabe, N. Shinoda, S. Okada and M. Iyo, 2003. Alterations on serum levels of brain-derived neurotrophic factor (BDNF) in depressed patients with or without antidepressants. Biol. Psychiatry, 54: 70-75.

22. Machado-Vieira, R., M.O. Dietrich, R. Leke, V.H. Cereser, V. Zanatto, F. Kapczinski, D.O. Souza, L.V. Portela and V. Gentil, 2007. Decreased plasma brain derived neurotrophic factor levels in unmedicated bipolar patients during manic episode. Biol. Physchiatry, 61: 142-144.

23. Branchi, I., I. D’Andrea, M. Fiore, V. Di Fausto, L. Aloe and E. Alleva, 2006. Early social enrichment shapes social behavior and nerve growth factor and brain-derived neurotrophic factor levels in the adult mouse brain. Biol. Psychiatry, 60: 690-696.
24. Miyazaki, K., N. Narita, R. Sakuta, T. Miyahara, H. Naruse, N. Okado and M. Narita, 2004. Serum neurotrophin concentrations in autism and mental retardation: A pilot study. Brain Devel., 26: 292295.

25. Nelson, K.B., J.K. Grether, L.A. Croen, J.M. Dambrosia, B.F. Dickens, J.L. Jelliffe, R.L. Hansen and T.M. Phillips, 2001. Neuropeptides and neurotrophins in neonatal blood of children with autism or mental retardation. Ann. Neurol., 49: 597-606.

26. Perry, E.K., M.L.W. Lee, C.M. Martin-Ruiz, J.A. Court, S.G. Volsen, J. Merrit, E. Folly, P.E. Iversen, M.L. Bauman, R.H. Perry and G.L. Wenk, 2001. Cholinergic activity in autism: abnormalities in the cerebral cortex and basal forebrain. Am. J. Phychiatry, 158: 1058-1066.

27. Pan, W., W.A. Banks, M.B. Fasold, J. Bluth and A.J. Kastin, 1998. Transport of brain-derived neurotrophic factor across the blood-brain barrier. Neuropharm., 37: 1553-1561.

28. Karege, F., M. Schwald and M. Cisse, 2002. Postnatal developmental profile of brain-derived neurotrophic factor in rat brain and platelets. Neurosci. Lett., 328: 261-264.

29. Nelson, P.G., T. Kuddo, E.Y. Song, J.M. Dambrosia, S. Kohler, G. Satyanarayana, C. VanDunk, J.K. Grether and K.B. Nelson, 2006. Selected neurotrophins, neuropeptides and cytokines: developmental trajectory and concentrations in neonatal blood of children with autism or Down syndrome. Int. J. Dev. Neurosci., 24: 73-80.

30. Gilmore, J.H., L.F. Jarskog and S. Vadlamudi, 2003. Maternal infection regulates BDNF and NGF expression in fetal and neonatal brain and maternal-fetal unit of the rat. J. Immunol., 138: 4955.

31. Gilmore, J.H., L.F. Jarskog and S. Vadlamudi, 2005. Maternal poly I:C exposure during pregnancy regulates TNF alpha BDNF and NGF expression in the neonatal brain and maternal-fetal unit of the rat. J. Neuroimmunol., 159: 106-112.

32. Borrell, J., J.M. Vela, A. Arevalo-Marin., E. Molina-Holgado and C. Guaza, 2002. Prenatal immune challenge disrupts sensorimotor gating in adult rats: Implications for the etiopathogenesis of schizophrenia. Neuropsychopharmacol., 26: 204-215.

33. Zuckerman, L., M. Rehavi, R. Nachman and I. Weiner, 2003. Immune activation during pregnancy in rats leads to a postpubertal emergence of disrupted latent inhibition, dopaminergic hyperfunction and altered limbic morphology in the offspring: a novel neurodevelopmental model of schizophrenia. Neuropsychopharmacology, 28: 1778-1789. 
34. Shi, L.M., Fatemi H., Sidwell R.W. and Ph. Patterson, 2003. Maternal influenza infection causes marked behavioral and pharmacological changes in the offspring. J Neurosci. 23: 297-302.

35. Fortier, M.E., R. Joober, G.N. Luheshi and P. Boksa, 2004. Maternal exposure to bacterial endotoxin during pregnancy enhances amphetamine-induced locomotion and startle responses in adult rat offspring. J. Psychiatr., Res., 38: 335-345.

36. Meyer, U., J. Feldon, M. Schedlowski and B.K. Yee, 2006. Immunological stress at the maternalfoetal interface: A link between neurodevelopment and adult psychopathology. Brain Behav. Immun., 20: 378-388.

37. Stadelmann, C., M. Kerschensteiner, T. Misgeld, W. Brück, R. Hohlfeld and H. Lassmann, 2002. BDNF and gp145trkB in multiple sclerosis brain lesions: neuroprotective interactions between immune and neuronal cells? Brain, 125: 75-85.

38. Vargas, D.L., C. Nascimbene, C. Krishnan, A.W. Zimmerman and C.A. Pardo, 2005. Neuroglial activation and neuroinflammation in the brain of patients with autism. Ann. Neurol., 57: 67-81.

39. Deumens, R., G.C. Koopmans, R.J.P. Jaken, K. Morren, T. Comhair, S. Kosar, H.W.M. Steinbusch, D. Bakker and E.A.J. Joosten, 2006. Stimulation of neurite outgrowth on neonatal cerebral astrocytes in enhanced in the presence of BDNF. Neurosci. Lett., 407: 268-273.

40. Kemper, T.L. and M. Bauman, 1998. Neuropathology on infantile autism. J. Neuropath. Exp. Neurol., 57: 645-652.

41. Fatemi, S.H., J. Earle, R. Kanodia, D. Kist, E.S. Emamian, P.H. Patterson, L.M. Shi and R. Sidwell, 2002. Prenatal viral infection leads to pyramidal cell atrophy and macrocephaly in adulthood: Implications for genesis of autism and schizophrenia. Cell Mol. Neurobiol., 22: 25-33.

42. Hertz-Picciotto, I., L.A. Croen, R. Hansen, C.R. Jones, J. Van de Water and I.N. Pessah, 2006. The CHARGE study: an epidemiologic investigation of genetic and environmental factors contributing to autism. Env. Health Persp., 114: 1119-1125.

43. Lord, C., A.M. Pickles, J. McLennan, M. Rutter, J. Bregman, S. Folstein, E. Fombonne, M. Leboyer and N. Minshew, 1997. Diagnosing autism: Analyses of data from the autism diagnostic interview. J. Autism Devel. Disord, 27: 501-517.

44. Lord, C., S. Risi, L. Lambrecht, E.H. Cook, B.L. Leventhal, P.C. DiLavore, A. Pickles and M. Rutter, 2000. The autism diagnostic observation schedule-generic: A standard measure of social and communication deficits associated with the spectrum of autism. J. Autism Devel. Disord., 30: 205-223.
45. Lord, C., B.L. Leventhal and E.H. Cook, 2001. Quantifying the phenotype in autism spectrum disorders. Am. J. Med. Genet., 105: 36-38.

46. Joseph, R.M., H. Tager-Flusberg and C. Lord, 2006. Cognitive profiles and social-communicative functioning in children with autism spectrum disorder. J. Child Psychol. Psychiatr. Allied Disciplines, 43: 807-821.

47. Croonenberghs, J., E. Bosmans, D. Deboutte, G. Kenis and M. Maes, 2002. Activation of the inflammatory response system in autism. Neuropsychobiol., 45: 1-6.

48. Jyonouchi H., S.N. Sun and H. Le, 2001. Proinflammatory and regulatory cytokine production associated with innate and adaptive immune responses in children with autism spectrum disorders and developmental regression. J. Neuroimmunol., 120: 170-179.

49. Ashwood, P. and A.J. Wakefield, 2006. Immune activation of peripheral blood and mucosal CD3(+) lymphocyte cytokine profiles in children with autism and gastrointestinal symptoms. J. Neuroimmunol., 173: 126-134.

50. Schulte-Herbruggen, O., C. Nassenstein, M. Lommatzsch, D. Quarcoo, H. Renz and A. Braun, 2005. Tumor necrosis factor- $\alpha$ and interleukin-6 regulate secretion of brain-derived neurotrophic factor in human monocytes. J. Neuroimmunol., 160: 204-209.

51. Aloe, L., F. Properzi, L. Probert, K. Akassoglou, G. Kassiotis, A. Micera and M. Fiore, 1999. Learning abilities, NGF and BDNF brain levels in two lines of TNF- $\alpha$ transgenic mice, one characterized by neurological disorders, the other phenotypically normal. Brain Res., 840: 125-137.

52. Heese, K., C. Hock and U. Otten, 1998. Inflammatory signals induce neurotrophin expression in human microglia cells. J. Neurochem., 70: 699-707.

53. Croll, S.D., C. Suri, D.L. Compton, Simmons M.V., G.D. Yancopoulos, R.M. Lindsay, S.J. Wiegand, J.S. Rudge and H.E. Scharfman, 1999. Brain-derived neurotrophic factor transgenic mice exhibit passive avoidance deficits, increased seizure severity and in vitro hyperexcitability in the hippocampus and entorhinal cortex. Neuroscience, 93: 1491-1506.

54. Nawa, H., M. Takahashi and P.H. Patterson, 2000. Cytokine and growth factor involvement in schizophrenia - support for the developmental model. Mol. Psychiatry, 5: 594-603.

55. Kurtzke, J.F., 1993. Epidemiological evidence for multiple-sclerosis as an infection. Clin. Micro. Rev., 6: 382-427.

56. Sospedra, M. and R. Martin, 2006. Molecular mimicry in multiple sclerosis. Autoimmunity, 39: 3-8. 